\title{
Obtaining an Adequate Specimen for the Diagnosis of Pigmented Lesions
}

\author{
Alyson Snyder, BS, Sara E. West, MD, Christopher M. Miles, MD, CAQSM, \\ and Steven R. Feldman, MD, PhD
}

\begin{abstract}
Melanoma is a common, potentially deadly disease but is curable if caught early. A specimen of a pigmented lesion must capture the entire width and depth of the lesion to evaluate the lesion's size, symmetry, and circumscription. Saucerization shaving, punching, and excision biopsy are 3 techniques that can be used to achieve a satisfactory specimen. Physicians should have a low threshold to biopsy a suspicious atypical pigmented lesion, but acceptable specimens are key in following through with an accurate diagnosis. (J Am Board Fam Med 2015;28:523-525.)
\end{abstract}

Keywords: Biopsy; Dermatology; Histology; Melanoma; Nevi, Melanocytic

Melanoma is a prevalent and potentially fatal disease but is curable if detected early. Identifying abnormal pigmented lesions and obtaining timely biopsies of lesions suspicious for melanoma are critical. Histologically adequate biopsy specimens are needed to make an accurate diagnosis. Inadequate specimens may delay, mislead, or even miss a diagnosis of melanoma. While only a small sample

This article was externally peer reviewed.

Submitted 31 January 2015; revised 14 February 2015; accepted 23 February 2015.

From the Center for Dermatology Research, Department of Dermatology (AS, SRF); the Department of Pathology (SEW, SRF); the Department of Public Health Sciences (SRF); and the Department of Family and Community Medicine (CMM), Wake Forest School of Medicine, WinstonSalem, NC.

Funding: The Center for Dermatology Research is supported by an unrestricted educational grant from Galderma Laboratories, L.P.

Conflict of interest: SRF is a speaker for Janssen and Taro and is a consultant and speaker for Galderma, Stiefel/GlaxoSmithKline, Abbott Labs, and Leo Pharma; Inc. SRF has received grants from Galderma, Janssen, Abbott Labs, Amgen, Stiefel/GlaxoSmithKline, Celgene, and Anacor. SRF is a consultant for Amgen, Baxter, Caremark, Gerson Lehrman Group, Guidepoint Global, Hanall Pharmaceutical Co. Ltd, Kikaku, Lilly, Merck \& Co. Inc., Merz Pharmaceuticals, Mylan, Novartis Pharmaceuticals, Pfizer Inc., Qurient, Suncare Research, and Xenoport. SRF is on an advisory board for Pfizer Inc. SRF is the founder of and holds stock in Causa Research, and holds stock in and is the majority owner of Medical Quality Enhancement Corp. SRF receives royalties from UpToDate and Xlibris.

Corresponding author: Steven R. Feldman, MD, PhD, Department of Dermatology, Wake Forest School of Medicine, Medical Center Blvd, Winston-Salem, NC 27157-1071 (E-mail: sfeldman@wakehealth.edu). of a tumor may be needed to identify the atypical cells and abnormal local growth pattern of a basal cell or squamous cell carcinoma, the histologic diagnosis of melanoma relies heavily on evaluation of the overall architecture of the melanocytic proliferation. It is our experience that inadequate incision specimens sometimes are submitted for evaluation.

Maize and Ackerman ${ }^{1}$ described 13 major histologic criteria for diagnosing melanoma (Table 1). The lesion's overall size, symmetry, and circumscription are major criteria for the diagnosis of pigmented lesions. ${ }^{1}$ For a pathologist to assess these criteria, a specimen must capture the entire breadth of the lesion. The entire width of the lesion may be captured by taking all the grossly visualized lesion. Critical diagnostic features at the edges of the specimen will be apparent if the specimen encompasses the entire width of the lesion and a small margin $(<1 \mathrm{~mm})$ of the surrounding skin.

A specimen must also be deep enough to determine the characteristics of the melanocytic dermal proliferation (Figure 1). Judgment guides how deep to biopsy; there may be no visible marker of how deep the tumor extends. Biopsy of a macular (flat) lesion of melanoma in situ does not need to be very deep, whereas a biopsy through all layers of the dermis should ensure an adequate depth for a thicker lesion. The depth of the tumor has prognostic significance; lesions $>4 \mathrm{~mm}$ thick have the poorest prognosis, so if the biopsy is $4 \mathrm{~mm}$ in depth 
Table 1. Histologic Criteria for Diagnosing Melanoma ${ }^{1}$

Breadth usually $>6 \mathrm{~mm}$

Asymmetry

Poor circumscription; atypical melanocytes beyond the most peripheral discrete nest of melanocytes within the epidermis

Increased number of single atypical melanocytes within the epidermis and epithelial structures of adnexa and, in some foci, single melanocytes predominate over nests of them

Melanocytes scattered in the upper reaches of the epidermis

Variation in the size and shape of nests of melanocytes

Nests of melanocytes are not equidistant

Irregular shapes of nests of melanocytes

Tendency of nests of melanocytes to confluence

Failure of maturation of atypical melanocytes, with progressive descent into the dermis

Asymmetrical, patchy distribution of melanin within the neoplasm

Extension of atypical melanocytes far down the epithelial structures of the adnexa

Asymmetrical distribution of inflammatory cell infiltrates of variable densities at the base of the neoplasm

but does not capture the full depth of the tumor, the poor prognosis is still readily apparent. Observed findings, experience, and judgment guide the operator's choice of depth. ${ }^{2}$

Three techniques can be used to biopsy a pigmented lesion based on the physician's judgment to achieve a satisfactory specimen and patient considerations: an excision, a saucerization shave, or a punch. An excisional biopsy involves complete removal of the entire lesion down to the fat, with a narrow margin of uninvolved skin. ${ }^{3}$ The saucerization shave technique (rather than a too-superficial shave) is also completely acceptable if it captures the full breadth and depth of the tumor. The width and depth of a saucerization shave are controlled by the operator and customized to the lesion being removed. When done adequately (going beneath the lesion), it captures all the histologic criteria that would be captured by an excisional biopsy. ${ }^{3}$ Shave biopsies have become increasingly popular because of their efficiency and cost considerations. A saucerization shave heals by secondary intention, does not require stitches, and generally leaves a flat to slightly raised scar. A punch biopsy can also provide the tissue necessary to evaluate a possible melanoma as long as the diameter of the punch used

Figure 1. Low-power magnification views of biopsied tissue. A: A presumably benign nevus. The section is of a punch biopsy extending into the deep dermis. The lesion in these sections seems to be small, symmetric, and well circumscribed. The melanocytes seem to be well nested, with no upper pagetoid extension, and they mature with descent into the dermis. The melanocytic proliferation extends to the lateral margins, however, so small size, symmetry, and circumscription are not ensured. B: A melanoma. This shave biopsy is a fully adequate specimen because it encompasses the full breadth and depth of the melanocytic proliferation. The proliferation is broad, asymmetric, and poorly circumscribed-characteristics diagnostic of melanoma. In addition, there are areas of randomly dispersed irregular nests of melanocytes, upward pagetoid extension by single melanocytes, and areas of confluence.

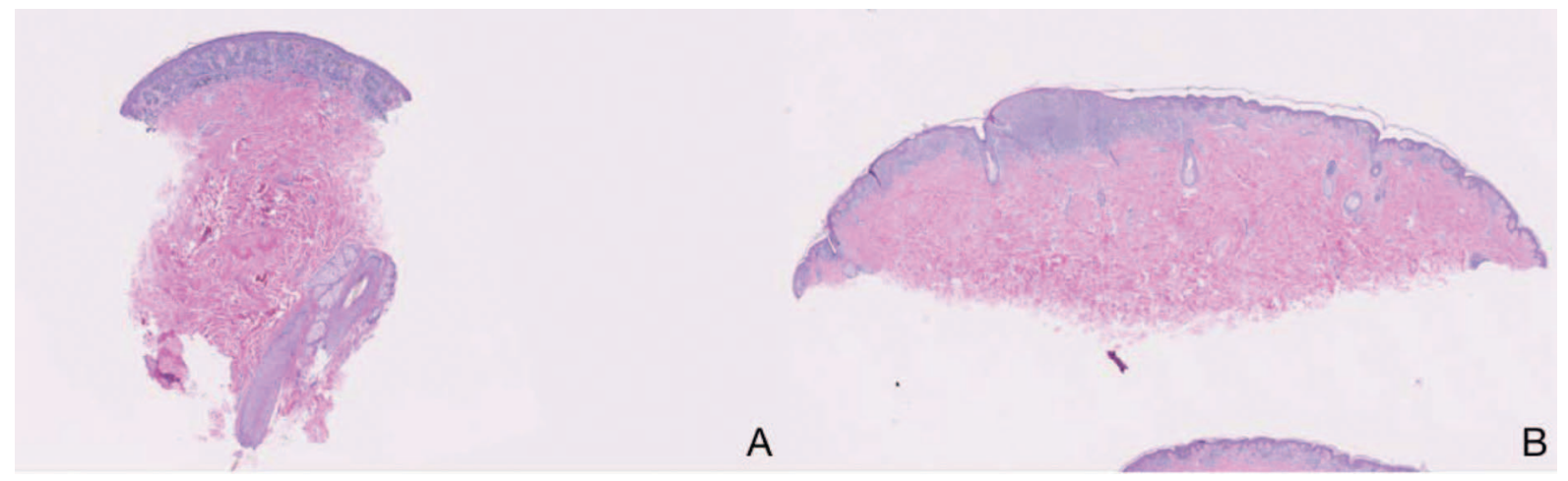


Figure 2. High-power magnification views of biopsied tissue. A: A presumably benign nevus. B: A benign-appearing section of melanoma. This area of the melanoma is small, symmetric, and well circumscribed, with small melanocytes that are well nested and located at the base of the rete, with no upper pagetoid extension. A punch biopsy from this area of the melanoma may have rendered an inaccurate benign diagnosis.

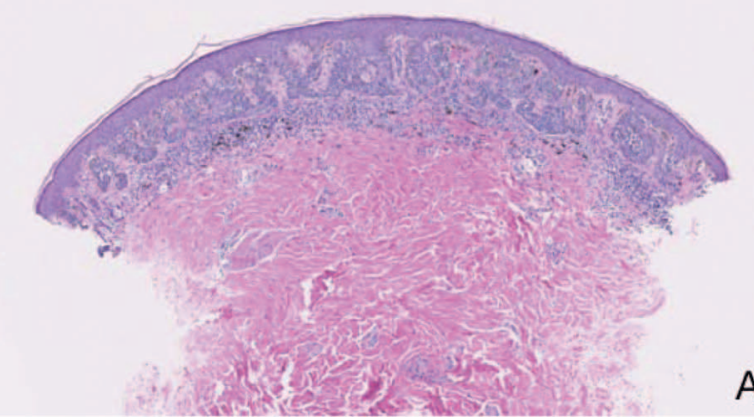

encompasses the entire lesion. Thus, one is limited to the diameters available. For example, a $6-\mathrm{mm}$ punch would be needed to biopsy a 5.5 -mm pigmented lesion. If a 6-mm punch is not available, then another biopsy technique should be chosen instead of taking a punch of only a portion of the pigmented lesion. Punches often are used if the lesion is expected to be deep. A shave or punch biopsy taken superficially or of only a small portion of the lesion can create a misleading histologic picture and ultimately cause an inaccurate diagnosis (Figure 2). For pictures and instructions of shave and punch biopsies see the article by Pickett. ${ }^{3}$

In addition to providing an adequate specimen, there needs to be a line of communication between the person performing the biopsy and the person interpreting the biopsy. Some clinical information provided to the pathologist-including whether the lesion has been previously biopsied and a clinical description-is helpful. If the entire lesion cannot be biopsied because of its size and/or location, the pathologist should be informed that the specimen represents a partial sample.

Breslow depth is the tumor thickness (measured in millimeters) from the top of the granular layer of the epidermis (or the base of an ulcer) to the deepest point of tumor invasion. ${ }^{4}$ The Breslow depth is the strongest prognostic factor for melanoma-related mortality. ${ }^{4}$ A superficial shave that does not capture the full depth of the tumor does not permit full determination of the Breslow depth, whereas a punch that is not sufficiently broad might not pro- vide sufficient material to evaluate the major histologic criteria for distinguishing benign from malignant melanocytic tumors.

A physician should have a low threshold to biopsy a suspected atypical pigmented lesion because early detection of melanoma leads to better outcomes. ${ }^{4}$ Saucerization shave, punch, and excisional biopsies are all acceptable techniques, provided they capture both the breadth and depth of the tumor. The decision of which technique to use depends on many factors, including the clinician's skill, pretest probability of melanoma, perception of lesion depth, lesion location, lesion size, equipment available, patient healing, and cosmetic results. If there is reluctance or an inability to perform an excision, doing an adequately deep and broad shave using a saucerization technique may be better. Superficial shaves or incisional punch biopsies of only small portions of pigmented lesions should be avoided to prevent histologic confusion and incorrect diagnoses.

\section{References}

1. Maize JC, Ackerman AB. Pigmented lesions of the skin: clinicopathologic correlations. Philadelphia: Lea \& Febiger;1987:182-3.

2. Tran KT, Wright NA, Cockerell CJ. Biopsy of the pigmented lesion-when and how. J Am Acad Dermatil 2008;59:852-71.

3. Pickett H. Shave and punch biopsy for skin lesions. Am Fam Physician 2011;84:995-1002.

4. Bolognia J, Jorizzo JL, Schaffer JV. Dermatology. 3rd ed. Philadelphia: Elsevier Saunders; 2012. 